\title{
Systemic Lupus Erythematosus Nephritis Class IV G
}

National Cancer Institute

\section{Source}

National Cancer Institute. Systemic Lupus Erythematosus Nephritis Class IV G. NCI

Thesaurus. Code C123119.

Systemic lupus erythematosus nephritis, characterized by active or inactive diffuse, global endo- or extracapillary glomerulonephritis that involves $50 \%$ or more of all glomeruli. (Weening, Jan J. et al. (2004). The Classification of Glomerulonephritis in Systemic Lupus Erythematosus Revisited. Journal of the American Society of Nephrology 15(2), 241-50.) 